\title{
DEVELOPMENT AND CHARACTERIZATION OF NOVEL MEDICATED HYDROGELS FOR WOUND DRESSING
}

\author{
Niladri Roy, Nabanita Saha, Takeshi Kitano, and Petr Saha
}

\begin{abstract}
$\square$ The medicated hydrogels were prepared aseptically under moist heat treatment using polyvinylpyrrolidone (PVP), sodium-carboxymethylcellulose $(C M C)$, polyethyleneglycol $(P E G)$, agar, glycerin and/or boric acid (BA) and designated as PVP-CMC and PVP-CMC-BA. The aim of this study was to develop some medicinal values-based hydrogels. BA was used to build up the medicinal values (antiseptic and antimicrobial properties) within the hydrogels. Optical images, scanning electron microscopy (SEM), and Fourier transform infrared (FTIR) spectroscopy of the hydrogels indicated that boric acid is uniformly dispersed within the cross-linked hydrogel network that may heal and protect the wounds from infections/sepsis. Swelling study in presence of water and physiological saline solution confirmed its reasonable absorption capacity. The rheological properties and mechanical properties demonstrated that the boric acid incorporated hydrogels are quite flexible. Assessment of antimicrobial property study proves that PVP-CMC-BA (3\% BA) has strong infection protection capacity.
\end{abstract}

Keywords Boric acid, Hydrogels, Swelling, Viscoelastic properties, Wound dressing

\section{INTRODUCTION}

The medicated hydrogels are the hydrogels that possess some medicinal value within them. They are cross-linked polymeric gels that have water- holding and nonadhering properties. Hydrogels can be prepared with monomers, prepolymers, or existing hydrophilic polymers as starting materials. They are generally specified by two component systems. One of the components is hydrophilic, which has water insoluble three-dimensional polymer networks, and the second component is water. They may swell in water up to a large extent and reach the equilibrium state while retaining their original shape (1). 
In fact, a hydrogel is considered as a kind of cheap, effective, convenient material for wound dressing, due to its good biocompatibility, water absorption ability, moisture retention, and ventilating capacity (2). They are closely resembled to living tissue in their physical properties because of their relatively high water content as well as soft and rubbery consistency (3). Numerous research studies have established that a moist wound environment is most favorable for healing (4). Hydrogel wound burn dressings were first invented by Rosiak et al. in 1989, which have many interesting properties, such as immediate pain control, easy replacement, transparency, absorption, prevention of loss of body fluids, barrier against bacteria, good adhesion, easy handling, oxygen permeability, control of drug dosage, and so on $(3,5,6)$.

The hydrogel dressings are usually directly applied to the wounds, thus the biocompatibility and non-toxicity factors are required to be taken into consideration. Polyvinylpyrrolidone (PVP) is a well-known polymer with good biocompatibility; hence, this polymer can be used as one of the main components of pseudo hydrogel preparation for temporary skin covers or wound dressings $(6,7)$. PVP is used in production of medicines and serves as blood substitute and blood detoxifier (8). PVP hydrogel itself does not exhibit good swelling properties but, when blended with polysaccharides such as carboxymethylcellulose (CMC) (2), carboxymethylchitosan (9), or other polysaccharides like agar or alginate, their swelling properties improve. Agar hydrogel is used as a poroelastic model that has similar hydration/dehydration properties simulating fluid and ion flow as that of the human skin (10). Agar acts as a natural cross-linking agent and its gelling characteristics provide mechanical reinforcement in hydrogels (11). Beside non-toxicity and biocompatible properties of polysaccharides, they have other advantages, for example, biodegradability and abundance in availability (2).

Boric acid (BA) is well known for its use as a home remedy to provide fast aid, for example: eye, ear, and skin treatments. It is also used in medicinal prescriptions because of its antiseptic, antifungal, as well as antibacterial properties. BA shows its preventive actions against skin problems such as athlete's foot and different kinds of fungal and yeast infections of the skin (12).Moreover, use of BA in the form of antiseptic cream [Boroline, Boroplus (2.5\% BA), B-Tex (10\% BA)] (13-15) is well known, but employing BA in hydrogel is a new concept in the domain of medicated hydrogels used for wound dressing. From the information in the literature, it gives assurance that the use of BA in health care products is safe up to a certain limit (16). There are permissible limits of boric acid content according to the purpose of use. The European Commission (EC) Directive 76/768/ EEC sets an upper limit of 5\% boric acid in talcs, $0.5 \%$ in oral hygiene products, and $3 \%$ in other products (17). 
In this article, we are reporting about the preparation technique of novel medicated hydrogels (i.e., PVP-CMC and PVP-CMC-BA). These hydrogels were developed by applying physical (moist heat sterilization) cross-linking technology followed by solution casting under aseptic environment. Already, different kinds of PVP- or PVA-based wound dressing hydrogels have been reported, but they were prepared by applying radiation cross-linking technology $(1,4,7)$. Many physical and chemical stimuli have been applied to induce responses of the smart hydrogel systems but for all time it is considered that physical stimuli are better than chemical stimuli. Moreover, it is essential to mention that physical stimuli included temperature, electric field, solvent, light, pressure, sound and magnetic field, and so on (18). Among all physical cross-linking methods, the radiation technology has become popular for the preparation of hydrogel dressing due to some advantages: there are no side products such as wastes, sewage, fumes, and all the components used to manufacture the hydrogel dressings are safe and human friendly (19); moreover, the radiation technology is comparatively inexpensive and does not need extra laboratory setup (4). Even though there are many advantages in radiation technology the area of radiation processing on natural polymeric material largely remain unexplored as most of the biopolymers are degraded when exposed to radiation (20). Still, the physical cross-linking methods like heating, drying, and irradiation are commonly applied to biopolymer (21). To overcome this drawback of irradiation cross-linking method on biopolymers, the moist heat treatment was applied (22) as the medicated hydrogel was prepared in combination with synthetic and natural biopolymers.

The main objective of this study was to produce hydrogel for health care that may also comprise the antiseptic and antimicrobial properties to improve the rate of healing of burns and wounds. The PVP-CMC hydrogels with and without BA were prepared and characterized and their physical appearance, physico-chemical structure, water uptake capacity, rheological, mechanical, and antimicrobial characteristics are described in this article.

\section{EXPERIMENTAL \\ Materials}

Polyvinylpyrrolidone K 30 (PVP: molecular weight 40,000), polyethylene glycol 3'000 (PEG: average molecular weight 3,015-3,685) and agar were obtained from Fluka, Switzerland; sodium carboxymethyl cellulose (CMC) was purchased from Sinopharm Chem. Reagent Co., Ltd, China; boric acid (BA) from Sigma-Aldrich, USA, and glycerin obtained from Lachema Ltd, Czech Republic. 


\section{Preparation of Medicated Hydrogels}

The ingredients used for the preparation of medicated hydrogels were PVP, CMC, BA, PEG, agar, and glycerin. Among the components present in the resultant hydrogel PVP and CMC function as base polymers, PEG performs as healing promoter (23), glycerin acts as a humectant (24), and agar serves as a gelling agent that can promote the formation of a hydrogel on cooling of the solution since the gel is formed due to the formation of helices and its association (25). BA acts as an antiseptic and an antimicrobial agent (26). It is easily mixed with the solution of PVP-CMC-PEG- Agar-Glycerin. The polymer solutions were prepared in sealed bottles. Then, after moist heat treatment $\left(15 \mathrm{lbs}, 120^{\circ} \mathrm{C}, 20 \mathrm{~min}\right)$ was applied it was followed by solution casting ( $20 \mathrm{ml}$ polymer solutions were poured) into Petri dishes ( $80 \mathrm{~mm}$ diameter). The mixture of polymer solution was then allowed to cool at room temperature $\left(20-22^{\circ} \mathrm{C}\right)$ under an aseptic environment to achieve the desired hydrogels. The hydrogel without BA was designated as "PVP-CMC" and the hydrogels with BA as "PVP- CMC- BA." The composition of the hydrogels is shown in Table 1 where the employed concentration of BA in hydrogels is within the permissible limit for medical application (0-4\%) according to the literature. Further, just after preparation of hydrogel the final product is designated as "fresh" or "before dry" and after drying termed as "dry" and finally, after swelling named as "swollen."

\section{Scanning Electron Microscopy (SEM)}

Hydrogels interior morphologies were evaluated by scanning electron microscopy (SEM) analysis (VEGA II LMU (TESCAN) operating in the high vacuum/secondary electron imaging mode at an accelerating voltage of 5-20 kV). The freeze dried samples of before dry PVP-CMC and PVP-CMC-BA hydrogels were analyzed. The hydrogels were frozen under $-81^{\circ} \mathrm{C}$ for 72 hours and then lyophilized (ALPHA 1-4 LSC, Labicom s.r.o, Czech Republic) for 24 hours. Thereafter, the samples were sputter coated with a thin layer of palladium/gold alloy to improve the surface conductivity and tilted 30 for better observation. The surface views as well as the cross-sectional views of the hydrogels were taken at magnification of 100x-10kx.

TABLE 1 Sample Index and Composition of Hydrogels (wt\%)

\begin{tabular}{|c|c|c|c|c|c|c|}
\hline Sairjple & PVil' & $\mathrm{OMC}$ & JPG & Mgar & Glyocrin & $B A$ \\
\hline PYPCMC & 0.2 & 0.8 & I & 2 & ] & 0 \\
\hline PVP-CMCBA & 0.2 & 0.8 & $t$ & 2 & I & $1-1$ \\
\hline
\end{tabular}




\section{Fourier Transform Infrared (FTIR) Spectroscopy}

Pure PVP, CMC, BA, and hydrogels of PVP-CMC and PVP-CMC-BA with 3\% BA (both in before dry and dry condition) were analyzed by FTIR, The ATR-FTIR spectroscopic analysis was conducted by using a NICOLET 320 FTIR Spectrophotometer with "Omnic" software package over the range 4,000-600 $\mathrm{cm}^{\text {"11 }}$ at room temperature. A uniform resolution of $2 \mathrm{~cm}^{-1}$ was maintained in all cases.

\section{Measurement of Water Absorptivity}

The degree of swelling could be described as water absorptivity of the hydrogel following Eq. (1) $(1,2 \mathrm{~V}, 28)$. The dry films of PVP-CMC and PVP-CMC-BA (0-4\% $\mathrm{BA}$ ) were obtained by drying the hydrogels at room temperature until they reached constant weight. The water absorptivity test was performed at room temperature. Small pieces $\left(1 \mathrm{~cm}^{2}\right)$ from the dry films were weighed and immersed in distilled water as well as in physiological saline solution, which contains $0.89 \% \mathrm{NaCl}$ (29). After the specified time intervals the water on the swollen gels was wiped off with tissue paper and the weights of specimens were determined. In each case, an average value of three samples has been represented in the graph to minimize error. The degree of swelling corresponds to the water absorptivity of the material, which is defined by Eq. (1) where $\mathrm{W}_{\mathrm{s}}$ and $\mathrm{W}_{\mathrm{d}}$ are weights of swollen gel and dried gel, respectively.

$$
\text { Water absorptivity } \left.(\%)=\mid\left(W_{s}-W_{d}\right) / W_{d}\right] \times 100
$$

\section{Measurement of Viscoelastic Properties}

The dynamic viscoelastic behavior of hydrogels of PVP-CMC and PVP- CMCBA (0-4\% BA) was investigated by using a parallel plate rheometer (ARES; Rheometries Scientific, USA) testing machine with an "RSI Orchestrator" software package. A $25 \mathrm{~mm}$ diameter parallel plate measuring geometry, with a gap of about $2-3 \mathrm{~mm}$ was used for the measurements under small strain amplitude (1\%) to maintain the measurements within the linear viscoelastic region (LVER). Dynamic frequency sweep tests were carried out at $28^{\circ} \mathrm{C}$ to observe the storage modulus $\left(\mathrm{G}^{\prime}\right)$ and loss modulus (G”) as a function of a wide range of angular frequencies (co 0.1 $100 \mathrm{rad} / \mathrm{s}$ ). In each case, three samples from the same composition were measured. 


\section{Measurement of Mechanical Properties}

The mechanical properties of both before dry and 30 and 60 min swollen samples were measured by an Instron model 8871, UK in compression mode at room temperature $\left(25^{\circ} \mathrm{C}\right)$ and $24 \%$ relative humidity with a load cell having a full-scale range of $1.0 \mathrm{KN}$. The before dry and swollen hydrogel samples $(25 \mathrm{~mm}$ diameter, 4-6 $\mathrm{mm}$ thickness ) were placed on the top plate of a compression load cell and compressed by a cylindrical-shaped metal disc at a constant crosshead speed of 0.5 $\mathrm{mm} / \mathrm{min}$ until the fracture of the hydrogels occurred. Compressive modulus $\mathrm{E}_{0}$ in the low range of compressive strain $(0-0.1 \mathrm{~mm} / \mathrm{mm}), \mathrm{E}$ in the middle range $(0.1-0.2 \mathrm{~mm} /$ $\mathrm{mm}$ ), compressive stress at fracture $\left\langle\mathrm{r}_{\mathrm{c}}\right.$ and compressive strain at fracture $7_{\mathrm{c}}$ were measured from the stress-strain curve and were used to represent the hydrogel mechanical property. The average value of two measurements for each sample was taken.

\section{Assay of Antimicrobial Property}

The antimicrobial properties of PVP-CMC and PVP-CMC-BA hydrogels were investigated by agar diffusion method $(30,31)$. Antimicrobial characteristics of these hydrogels were examined on the basis of the dimension of inhibition zone generated in the presence and absence of pathogenic bacteria like Escherichia coli, Staphylococcus aureus, Klebsiella pneumoniae, and Pseudomonas aeruginosa and fungi like Candida albicans and Candida parapsi- losis.TYve antibacterial assay was conducted in a sterile nutrient agar (2\%) medium that was inoculated with the aforementioned bacterial strains under aseptic condition. After solidification of nutrient agar, a piece of hydrogel (15 $\mathrm{mm}$ diameter) was placed on the surface of the medium. The testing plates were then incubated in a temperature-controlled incubator at $37^{\circ} \mathrm{C}$ to observe the effect of PVP-CMC and PVP-CMC-BA hydrogels on the growth of individual bacteria. The antifungal assay was conducted at $30^{\circ} \mathrm{C}$ using malt extract agar $(2 \%)$ medium in the same manner using the procedure described earlier.

\section{RESULTS AND DISCUSSION}

\section{Optical View}

Figure 1 shows the physical appearance (optical view) of the medicated hydrogels of PVP-CMC and PVP-CMC-BA before and after drying. It can be seen from Fig. 1 (a) and (b) that during the fresh state (before drying) both the hydrogels PVP-CMC and PVP-CMC-BA look similar in shape, size, and texture (i.e., round, whitish, semitransparent, soft rubber- like). But, when 

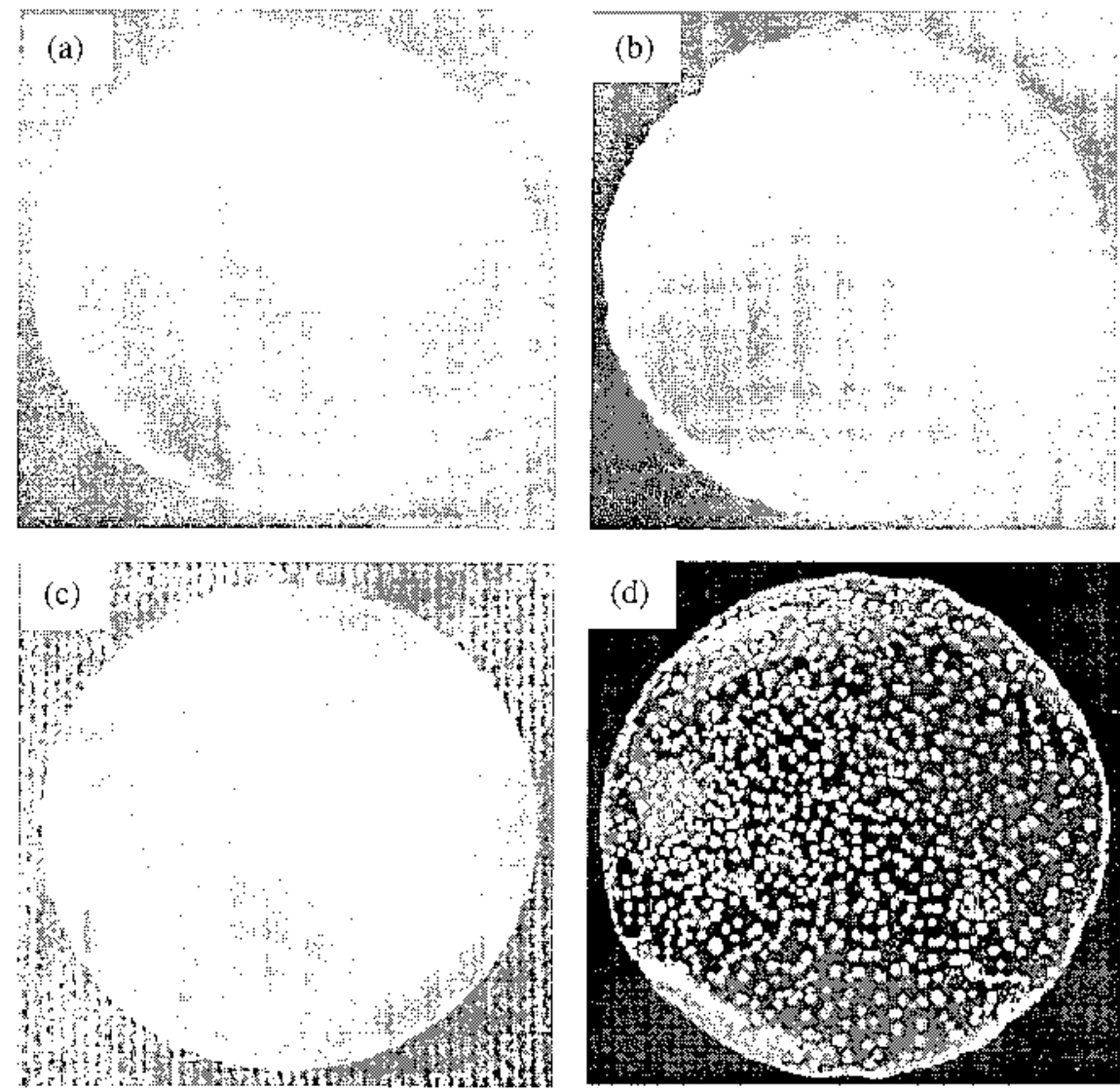

FIGURE 1 Optical view of medicated hydrogels PVP-CMC (a, before drying and c, dry) and PVP-CMC- BA (b, before drying and d, dry).

they are dried, their appearance is changed as shown in Fig. 1 (c) and (d). After drying the PVP-CMC hydrogel becomes almost like a thin, transparent, and flexible film but maintaining its own shape. On the other hand, the presence of BA is clearly visible as white dots all over the film of PVP- CMC-BA although other characteristics are not very much affected. The appearance of white dots, which exhibits the presence of $\mathrm{BA}$ in hydrogel, varies according to the concentration of BA\%. Boric acid (BA) is white crystalline solid material. It is soluble in hot water up to a certain limit. In our case, the polymer solution with BA is prepared under moist heat condition with $3 \% \mathrm{BA}$, which is easily dissolved in the solution. As a final product of hydrogel (before drying), it contains more than $90 \%$ of water, so BA remains dissolved in water and forms some hydrogen bonds too. But, when the hydrogel is allowed to dry, the water evaporates during drying process 
and the BA reaches to its saturation and re-crystallizes, which appears as white dots. The optical view of the medicated hydrogels has been examined to understand the changes in physical appearance between fresh (before drying) and dry states.

\section{SEM Micrographs}

Figure 2 represents the interior morphologies of medicated hydrogels PVP-CMC and PVP-CMC-BA (3\% BA). Figure 2 (a) and (b) show the surface and crosssectional SEM views of PVP-CMC and Fig. 2 (c) and (d) show the surface and crosssectional SEM views of PVP-CMC-BA, respectively. From all the images, it is clearly understandable that both PVP-CMC and PVP-CMC-BA blend materials are hydrogels having three- dimensional network structure. In comparison to PVP-CMC, PVP-CMC-BA contains denser porous-network structure (Fig. 2 (c) and (d)). This difference in morphology between the two hydrogels could be explained as the influence of BA. Here, BA is performing the role of partial cross- linking agent, which causes denser cross-linking network structure in PVP-CMC-BA.

\section{FTIR Spectroscopy}

FTIR spectra of pure BA, PVP, CMC and fresh hydrogels of PVP-CMC and PVP-CMC-BA are shown in Fig. 3 (a). The FTIR spectra of other components are not mentioned for the sake of simplicity. Some characteristic peaks in PVP spectrum can be ascribed as: $1,281 \mathrm{~cm}^{-1}$ for $\mathrm{C}-\mathrm{N}$ stretching vibration, $1,658 \mathrm{~cm}^{-1}$ for $\mathrm{C}=\mathrm{O}$ vibration (2). In the case of PVP-CMC hydrogel, the $1,658 \mathrm{~cm}^{-1}$ peak of PVP, and $1,588 \mathrm{~cm}^{-1}$ peak of CMC become a single broad peak $\left(1,628 \mathrm{~cm}^{-1}\right)$. This suggests that there is cross-linking of CMC or grafting of CMC and other ingredients of hydrogels are linked with PVP chains in PVP-CMC blend matrix. Pure BA shows two large peaks at $1,401 \mathrm{~cm}^{-1}$ and $1,189 \mathrm{~cm}^{-1}$ (Fig. 3 (a)). Subsequently, to compare the interaction of BA with the PVP-CMC blend matrix, FTIR spectra of PVP-CMC and PVP-CMC-BA (both in before drying and dry) are represented in Fig. 3 (b). From these FTIR spectra of hydrogels presented in Fig. 3 (b), it is impossible to justify whether there is some interaction of BA with PVP-CMC polymer blend as there is no significant new peak appeared in PVP-CMC-BA hydrogel, neither in before dry nor in dry state. Only, the existence of BA in PVP-CMC-BA hydrogel is established by FTIR study. The peak characteristic of BA at $1401 \mathrm{~cm}^{-1}$ and $1189 \mathrm{~cm}^{-1}$ appears almost at the same position in the FTIR spectra PVP-CMC-BA as of pure BA despite the intensity of peaks are reduced. 

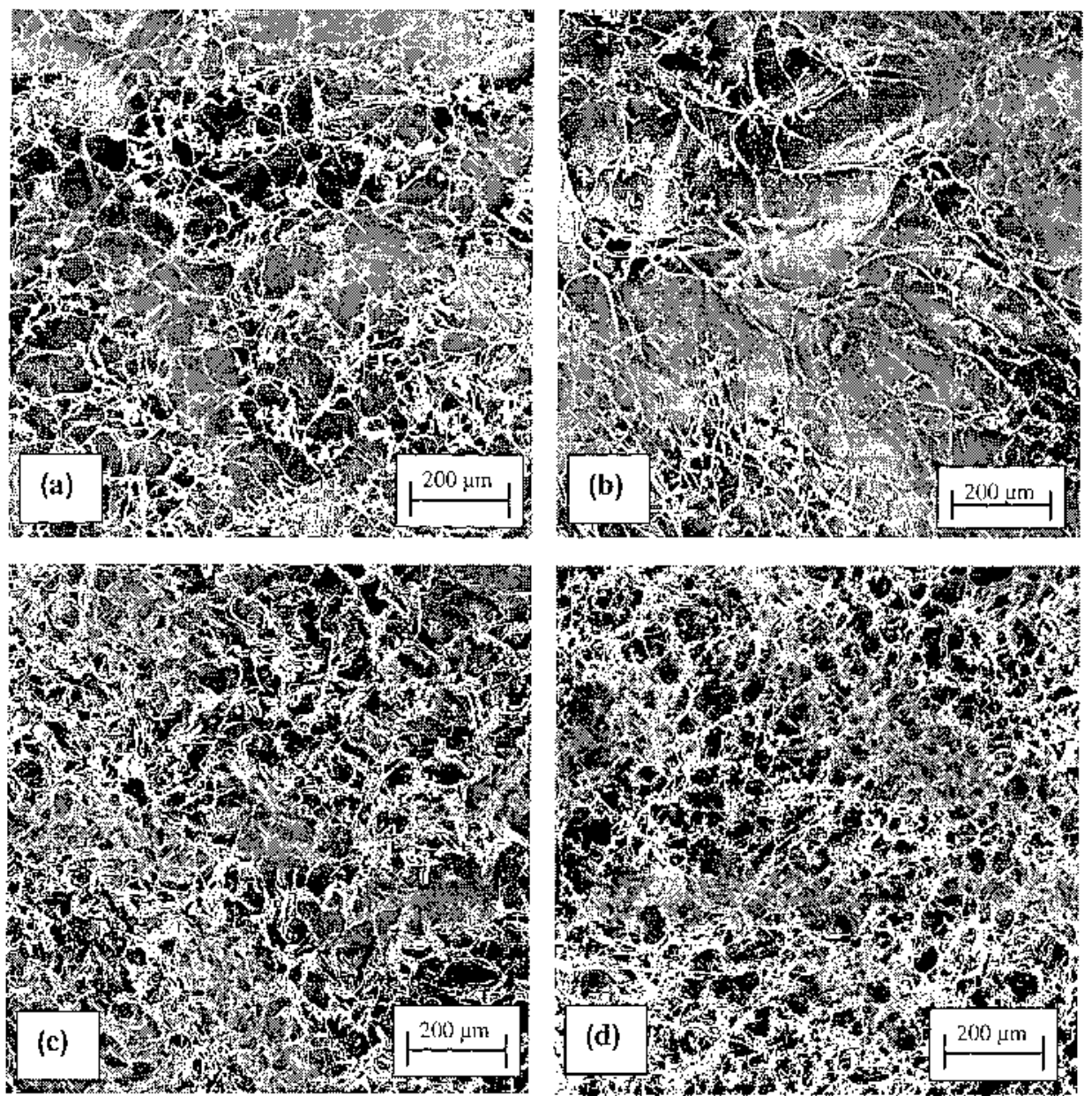

FIGURE 2 SEM image of freeze dried medicated hydrogels (a) PVP-CMC (surface view), (b) PVP-CMC (cross-sectional view), (c) PVP-CMC-BA (surface view), and (d) PVP-CMC-BA (cross-sectional view).

\section{Factors Affecting on Swelling}

One of the main characteristics of wound dressing hydrogels is the capacity to absorb fluids (blood plasma or serum) from wounds; thus, it is important to know the factors affecting the swelling behavior and degree of swelling of any newly developed hydrogels. Fluid absorption capacity also depends on composition of hydrogel, external physical influences like temperature, $\mathrm{pH}$, time, types of fluids, and so on. The ability to swell and the extent of swelling of hydrogels are mainly governed by two factors: the hydrophilicity of its polymer chains and the crosslinking density (2). Swelling behavior of the hydrogels was measured using distilled water instead of 


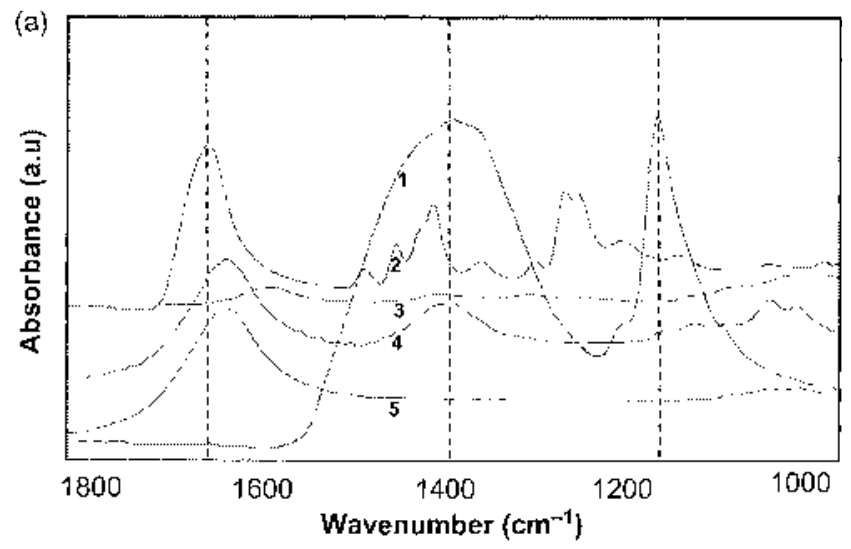

FIGURE 3 (a) FTIR spectra of pure (1) BA, (2) PVP, (3) CMC, (4) PVP-CMC-BA (3\%, before drying), (5) PVP-CMC (before drying).

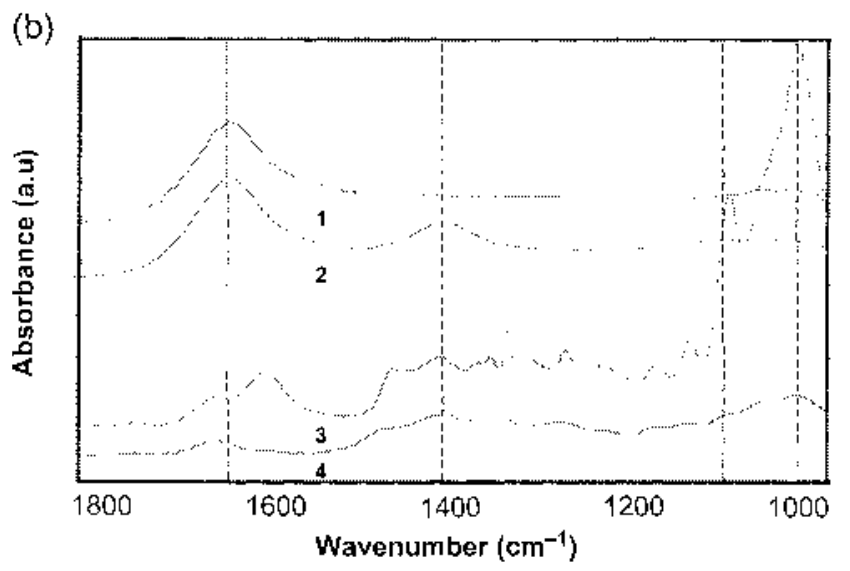

FIGURE 3 (b) FTIR spectra of (1) PVP-CMC (before drying), (2) PVP-CMC-BA (3\%, before drying, (3) PVP-CMC (dry), (4) PVP-CMC-BA (3\%, dry).

blood (as blood plasma contains about $90-92 \%$ of water) and in physiological saline solution $(0.89 \% \mathrm{NaCl})$, whose osmotic pressure is approximately equal to that of human tissue fluid $(32,33)$. The swelling behavior of dry PVP-CMC and PVP-CMCBA hydrogels in distilled water and in physiological saline solution is represented in Fig. 4. It can be seen from this figure that types of fluid and composition of hydrogel have great influence on degree of swelling. PVP-CMC hydrogel always shows the higher degree of swelling compared to PVP-CMC-BA. The noticeable difference in water uptake capacity was because of the incorporation of BA in hydrogel and salt concentration 


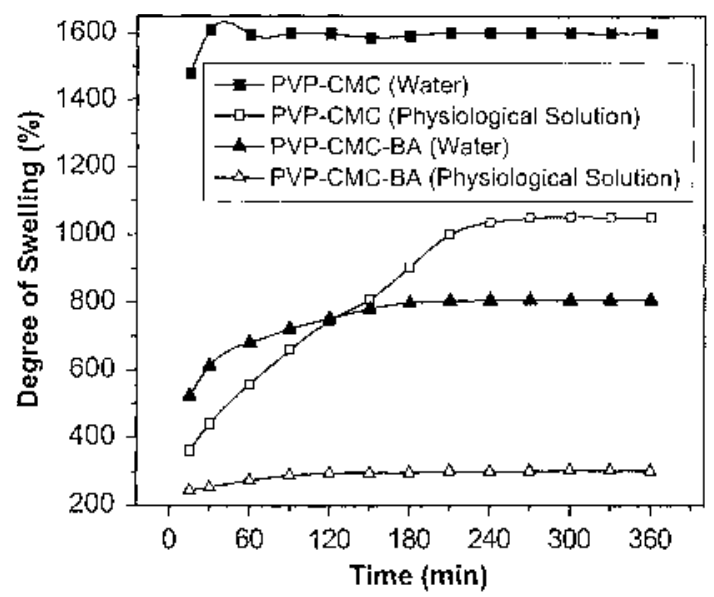

FIGURE 4 Swelling behavior of PVP-CMC and PVP-CMC-BA hydrogels (dry) in distilled water and in physiological saline solution.

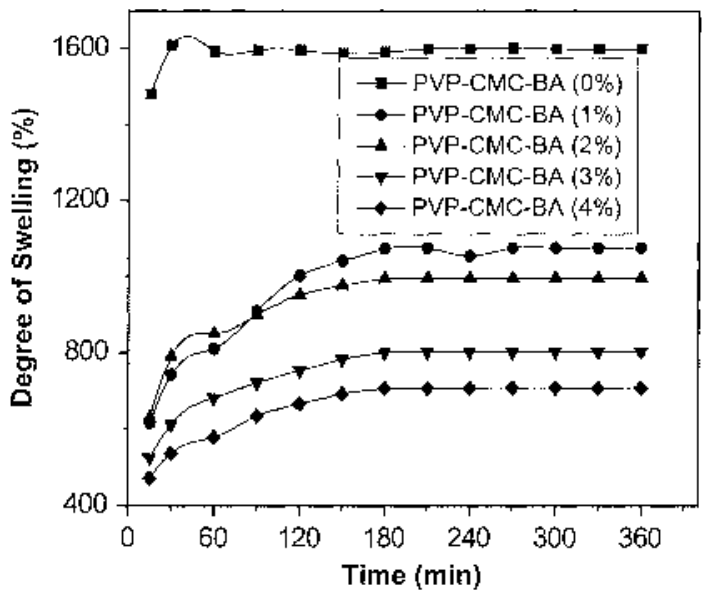

FIGURE 5 Effect of BA on swelling behavior of PVP-CMC-BA hydrogels (dry) in distilled water.

gradient in fluids. BA increases the cross-linking density in PVP-CMC-BA, which in turn decreases the swelling property of the hydrogels.

Figure 5 represents the effect of BA on the swelling behavior of dry PVP- CMCBA hydrogels in distilled water. The swelling study was performed up to $360 \mathrm{~min}$ and the result showed that the PVP-CMC-BA hydrogel with 0\% BA demonstrated significantly higher water absorption property than the PVP-CMC-BA (1-4\% BA) hydrogels. In each case, the swelling property of 
the hydrogels increases with time. The PVP-CMC hydrogel without BA reaches to equilibrium condition within $90 \mathrm{~min}$, whereas in the case of each PVP-CMC-BA hydrogel the degree of swelling value reaches to equilibrium condition after around $180 \mathrm{~min}$. On the other hand, it is noticeable that the degree of swelling decreases gradually with the increasing concentration of BA in hydrogels. That may be explained as the concentration of BA increases; it creates more cross-linking into the hydrogels, which causes lowering in degree of swelling. Because some amount of intermolecular hydrogen bonding of this component is broken by water, intramolecular hydrogen bonds are formed in appropriate sequences together with cross- linking with boric acid (34).

The swelling behavior of the polymeric hydrogels is based on the theory that there is a network of chains of polyelectrolyte containing ionizable groups, and the mobile counterions in the gels develop a large swelling pressure due to some intermolecular non-covalent interaction, such as cou- lombic repulsion, hydrogenbonding, and polar forces $(2,35)$. Thus, the polymeric interactions increase and cause a very high sorption rate.

\section{Viscoelastic Properties}

Viscoelasticity denotes the combination of viscous and elastic properties in a material with the relative contribution of each being dependent on time, temperature, stress, and strain rate (36). It has been reported that the viscoelastic properties of hydrogels correlate strongly with their microstructures and can provide useful information for modulating strongly with their performance characteristics $(36,37)$. Moreover, in the swollen state the mass fraction of water in a hydrogel is much higher than the mass fraction of the polymer (2), which may affect the rheological behavior of hydrogels in the swollen state. In the PVP-CMC-BA hydrogel, BA plays significant roles in the absorption of secreted fluid from the wound, maintaining the elasticity of the hydrogels besides inhibiting the pathogens, which is one of its usual characteristics. Thus, the effect of BA on viscoelastic properties of PVP-CMC-BA has been evaluated.

Figure 6 represents the effect of BA concentration (0-4\%) on storage modulus $\left(\mathrm{G}^{5}\right.$, unfilled symbols), which denotes elastic property and loss modulus (G", filled symbols) which represents viscous property of hydrogels with respect to angular frequency $(u>)$ for fresh samples. From Fig. 6, it is clear that concentration of BA in hydrogel also has a significant influence on rheological properties. The hydrogel without BA shows the lowest values in G' and G', but the values of G' and G' increase as the concentration of BA increases up to $3 \%$ and then starts to decrease, which may be due to the action of residual BA, which acts as a lubricant. This indicates that the PVP-CMC-BA with $3 \% \mathrm{BA}$ is the most elastic among the other BA-based hydrogels. 


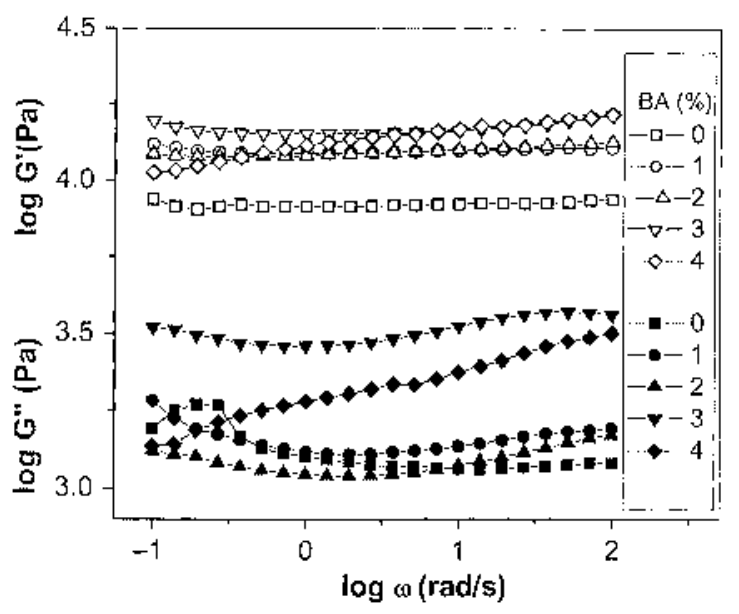

FIGURE 6 Effect of boric acid (BA) concentration (0-4\%) on storage modulus (G\ unfilled symbols) and loss modulus (G", filled symbols) for fresh PVP-CMC-BA hydrogels (before drying) with respect to angular frequency $(\boldsymbol{u})$.

From the aforementioned explanation, it is understandable that 3\% BA will be ideal for PVP-CMC-BA hydrogel preparation. Thus, the rest of the investigations were carried out using PVP-CMC-BA hydrogel with 3\% BA.

It is expected that when a material absorbs water or any liquid its property is changed. Similarly, when a dry hydrogel is absorbing water it starts to swell and its viscoelastic property changes gradually with time. As mentioned before, the storage modulus (G') provides information about elastic property and loss modulus (G”) gives information about viscous property of hydrogel. Fig. 7 shows the comparative effect between two hydrogels: PVP-CMC and PVP-CMC-BA with 3\% BA of their state (fresh/before dry and after swelling) on G' (unfilled symbols) and G" (filled symbols) with respect to angular frequency $(U J)$. In each and every state of the samples of PVP-CMC and PVP- CMC-BA, the values of G' are higher than G" in the whole range of angular frequency. Figure 7 (a) shows viscoelastic properties of the fresh hydrogels, whereas Figs. 7 (b-d) displays the viscoelastic properties of the same hydrogels when they are swelled in distilled water from dry state for 15, 30, and 60 min. From Fig. 7 (a), it is clearly visible that both the hydrogels are elastic in nature. PVP-CMC-BA is more elastic than PVP-CMC because incorporation of BA is responsible to develop more cross-linking in the hydrogels.

From Figs. 7 (b-d), it is clear that after swelling the elastic moduli (G') are lowered for both hydrogels. PVP-CMC-BA still keeps up its predominating rubbery nature as G' are about one decade higher than G'. But, in the case of PVP-CMC, after swelling G" values reach near to the G' values, shifting toward viscous nature. During swelling some bonds in the hydrogel 

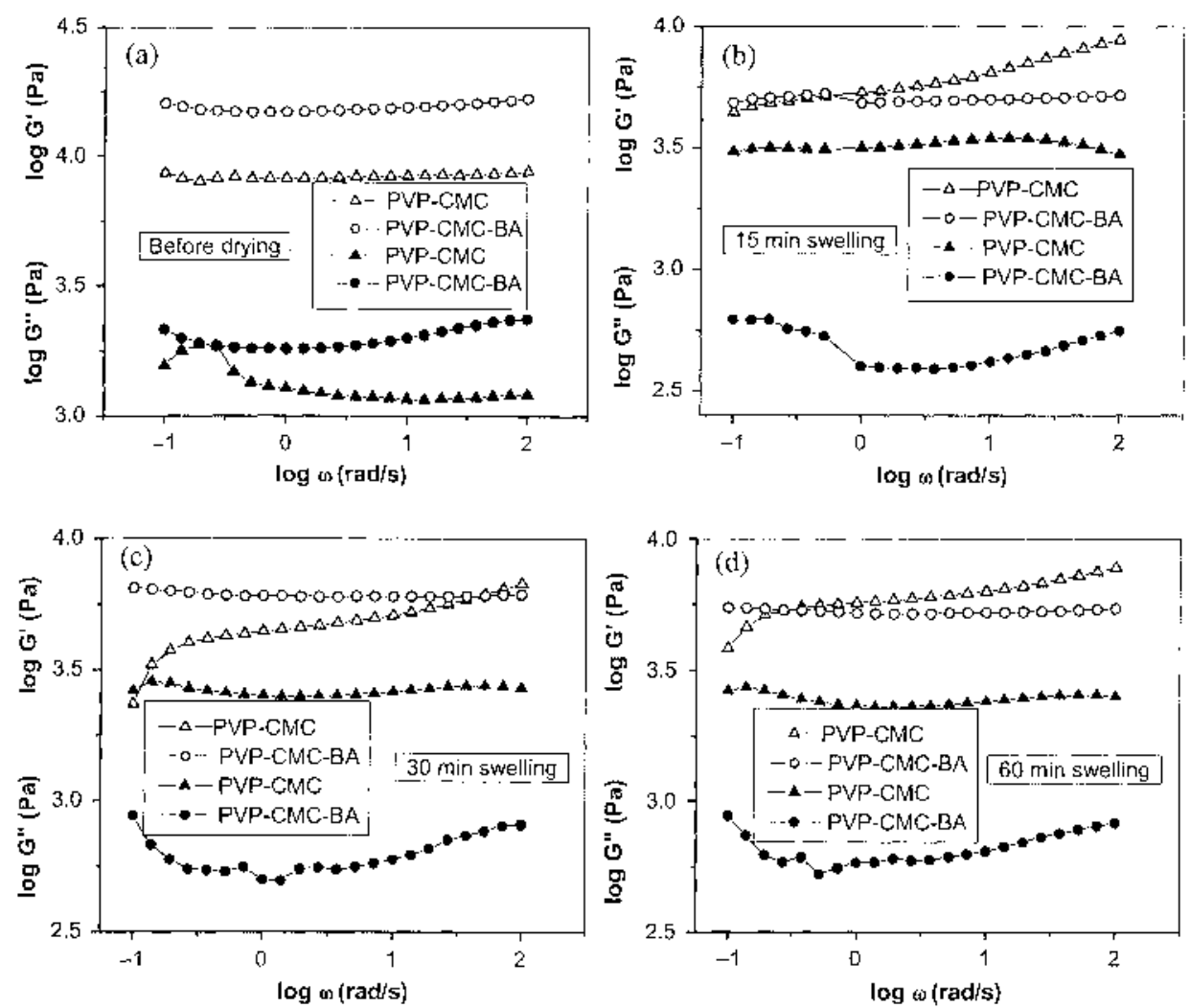

FIGURE 7 Effect of state, composition of hydrogels (PVP-CMC and PVP-CMC-BA with 3\% BA), and time of swelling on storage modulus ( $\mathrm{G} \backslash$ unfilled symbols) and loss modulus (G", filled symbols) with respect to angular frequency (a) hydrogels before drying and hydrogels after swelling, (b) $15 \mathrm{~min}$, (c) $30 \mathrm{~min}$, (d) $60 \mathrm{~min}$

cross-linking network are broken by hydration energy for both the hydrogels, and also BA starts to diffuse from hydrogels in water. So the G' values decrease after swelling. The presence of BA in PVP-CMC-BA helps the hydrogel to remain still elastic after swelling, whereas more structural changes occur for PVP-CMC. From these Figs. 7 (b-d), it is clear that swelling time does not affect much on the viscoelastic behavior of PVP-CMC and PVP-CMC-BA hydrogels. These types of curves are typical for gels, which have been also observed for other types of gels (36, 38).

Furthermore, there are several factors that affect the viscoelastic property of gel, such as the composition and the concentration of the dispersed materials and the lag time between preparation and measurement (39). As a whole, the viscoelastic behavior of the gels depends strongly on the mechanism of gel formation. The formation of the three-dimensional network structure and its mechanical property is influenced by covalent bonding, crystallization, and 
molecular secondary forces like hydrogen bonding, molecular entanglements, and hydrophobic interactions (37). From this point of view of storage and loss moduli, where the dependence of swelling time and angular frequency on both moduli are different, it is considered that the influence of the mechanism of structural change by the reduction of cross-linking density with the existence of BA is also different in case of both moduli.

\section{Mechanical Properties}

The comparison of mechanical properties between fresh (before drying) and 30 and 60 min swollen samples of PVP-CMC and PVP-CMC-BA hydrogels are shown in Table 2. The initial thickness of before drying hydrogel samples was $6 \mathrm{~mm}$. These $6 \mathrm{~mm}$ thick hydrogels were dried and used to investigate the effects of swelling after 30 and 60 min on compressive properties of hydrogel. It can be seen from Table 2 that in case of before drying samples the compressive modulus $\mathrm{E}_{0}$ and $\mathrm{E}$ of PVPCMC-BA is higher than those values of PVP-CMC. But, after swelling (30 and 60 min) PVP-CMC hydrogel shows higher values of both $\mathrm{E}_{0}$ and $\mathrm{E}$ than those of PVPCMC-BA. These results can be explained this way; BA is responsible for the development of more cross-linking in hydrogel as it acts as a partial cross- linking agent too. Those condense cross-linking structures (Figs. 2 (c) and

(d) ) influence to exhibit better mechanical properties in before drying samples of PVP-CMC-BA. But, when the thick hydrogels (about $6 \mathrm{~mm}$ ) are in progress to swell after drying, BA starts to diffuse from the surface, but not diffuse from the inner part of the hydrogel completely within the observed swelling time (30 and $60 \mathrm{~min}$ ) because PVP-CMC-BA hydrogel is not swelled up to its equilibrium condition. For this reason, it is impossible to determine the actual mechanical properties of swollen PVP-CMC-BA hydrogel. An example of compressive behavior of PVP-CMC-BA is shown in Fig. 8 by plotting a stress-strain curve up to fracture.

TABLE 2 Mechanical Properties of PVP-CMC and PVP-CMC-BA Hydrogels

\begin{tabular}{|c|c|c|c|c|c|c|}
\hline \multirow[b]{3}{*}{ Mechanicell paralneter } & \multicolumn{3}{|c|}{ PVP-CMC } & \multicolumn{3}{|c|}{ PN-CMC-BA } \\
\hline & \multirow{2}{*}{$\begin{array}{l}\text { Belore } \\
\text { drying }\end{array}$} & \multicolumn{2}{|c|}{ Sivollen } & \multirow{2}{*}{$\begin{array}{l}\text { Betorc } \\
\text { drying }\end{array}$} & \multicolumn{2}{|c|}{ Swollen } \\
\hline & & $30 \mathrm{~min}$ & $60 \mathrm{~min}$ & & 30 min & 60 min \\
\hline $\begin{array}{l}\text { Comprossive modulus } \mathrm{F}_{0} \text { at }(\mathrm{O}-0.1 \mathrm{~mm} / \mathrm{mm} \\
\left.\text { strain ( } \mathrm{M} \mathrm{P}_{\mathrm{n}}\right)\end{array}$ & 0.1025 & 0.1765 & 0.12495 & 0.1215 & 0.1250 & 0.0700 \\
\hline 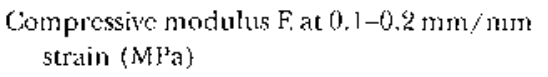 & 0.1285 & 0.3473 & 0.2825 & 0.1965 & 0.2750 & 0.1625 \\
\hline Comprossive stress $\sigma_{0}$ at fracture $(\mathrm{HA} \mathrm{a})$ & 0.0995 & 0,0650 & 0.0417 & 0.0250 & 0.0700 & 0.0425 \\
\hline Compressive strain y at fracture (mosin/min) & 0.2150 & 0.1682 & 0.1810 & 0.1585 & 0.2400 & 0.2875 \\
\hline
\end{tabular}




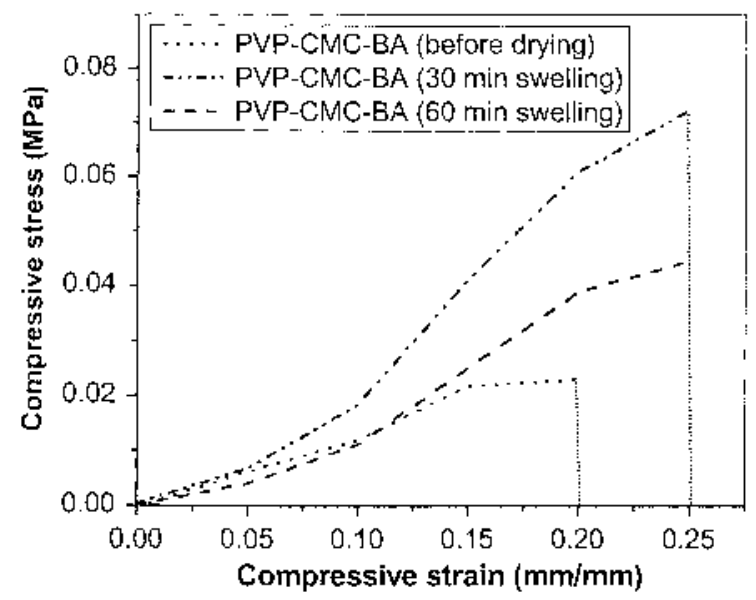

FIGURE 8 Mechanical property of before drying and 30 and 60 min swelled PVP-CMC-BA hydrogel.

\section{Antimicrobial Property}

The antimicrobial property of hydrogel is necessary to be determined before recommending the hydrogels for health care application as the presence of pathogenic microbes like bacteria (Staphylococcus aureus, Escherichia coli, Pseudomonas aeruginosa, and Klebsiella pneumonia) and fungi (Candida albicans and Candida parapsilosis), which are quite common on wound infection, wound burn, or on sudden injuries. Fig. 9 shows antibacterial effects of the hydrogels on various bacteria: (a) Staphylococcus aureus, (b) Escherichia coli, (c) Pseudomonas aeruginosa, and (d) Klebsiella pneumonia,whereas, Fig. 10 represents the antifungal effects on fungi like (a) Candida albicans and (b) Candida parapsilosis through the formation of zone of inhibition. It can also be seen from Figs. 9 and 10 that PVP-CMC hydrogel does not have antimicrobial effect but PVP-CMC-BA hydrogel shows strong antimicrobial effect in the presence of both bacteria and fungi. BA shows significant influence in achieving antimicrobial property within PVP-CMC-BA hydrogel. Incorporation of BA (3\%) in hydrogel is proven to be an effective concentration to develop antimicrobial property in the hydrogel, which comes within the permissible limit from the health care point of view (16).

\section{CONCLUSIONS}

The objective of the study was to develop a novel medicated hydrogel dressing based on boric acid. Incorporation of BA, having antiseptic, antibacterial, and antifungal properties, in hydrogel is an innovative attempt 

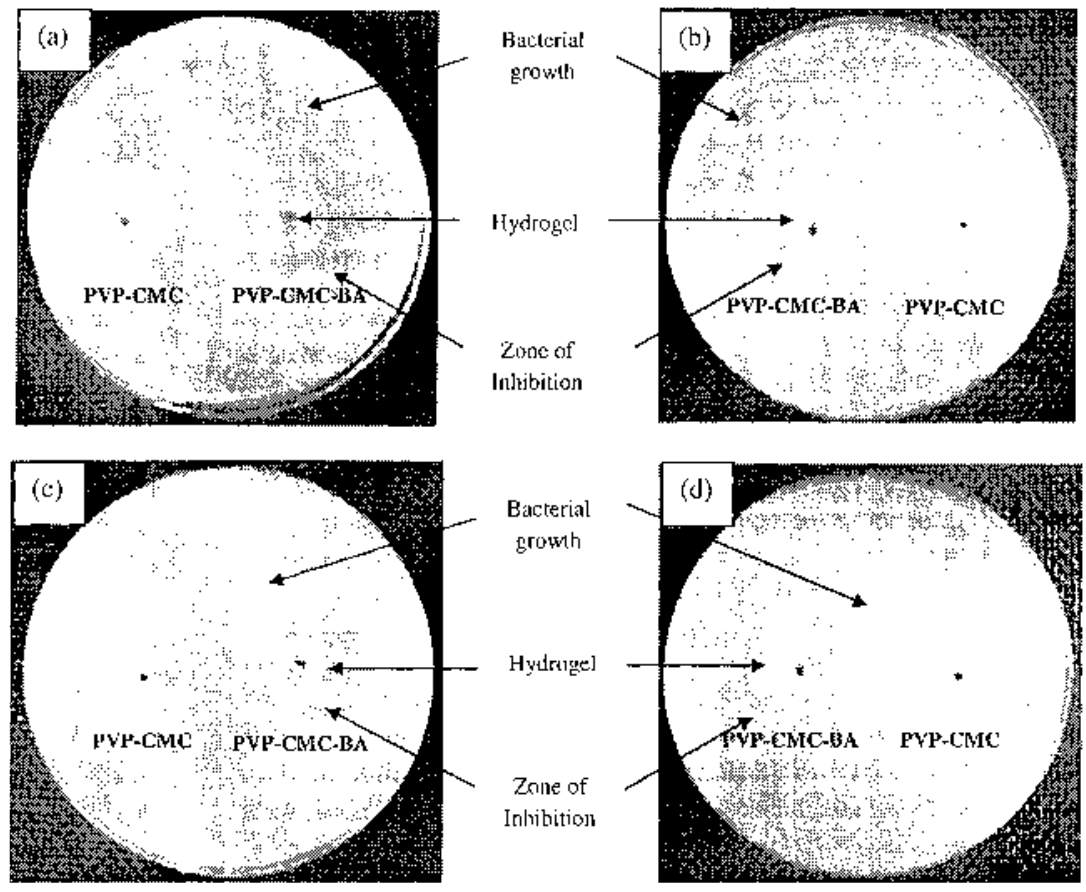

FIGURE 9 Image of antibacterial effects of PVP-CMC hydrogels without and with 3\% boric acid (BA) in presence of skin infection causing common bacteria; (a) Staphylococcus aureus, (b) Escherichia coli, (c) Pseudomonas aeruginosa, (d) Klebsiella pneumoniae.
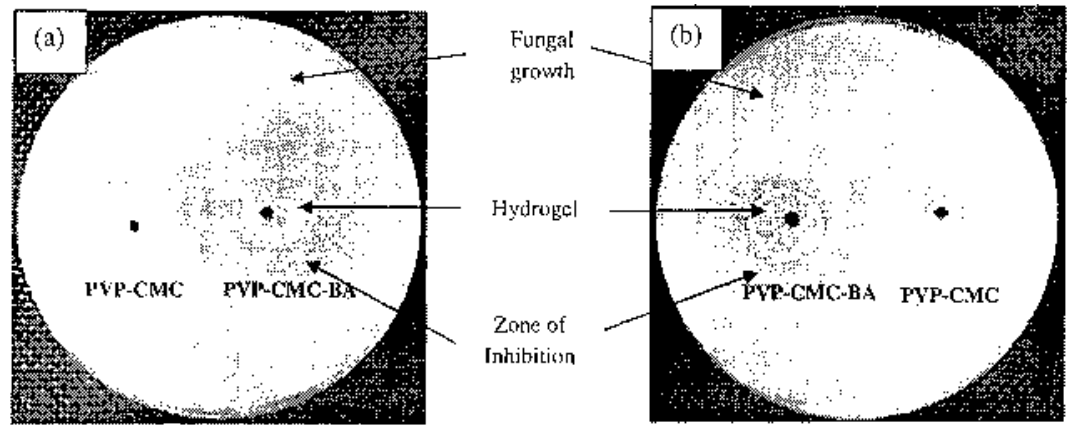

FIGURE 10 Image of antifungal effects of PVP-CMC hydrogels without and with $3 \%$ boric acid (BA) in presence of skin infection causing common fungi; (a) Candida albicans And (b) Candida parapsilosis.

for application in medical devices. Hydrogel with $3 \%$ BA has been selected considering its permissible limit of dose as a medicine (16). From the experimental results of SEM microstructure, swelling property, viscoelastic, and mechanical properties, it was confirmed that BA in current work not 
only acts as an antimicrobial agent but also fulfills partially the role of a "crosslinker." It can be assumed that the acid group (-COOH) of CMC may react with hydroxyl group (-OH) of boric acid and glycerin, as a result form the chelate structure through ester linkages, which exhibits the dense porous network structure. The PVP-CMC hydrogel having the permissible limit of boric acid (3\%) for health care application, demonstrated quite a good antimicrobial property in presence of skin infection causing bacteria and fungi, thus, this hydrogel could be recommended for its appliance as a wound dressing material to protect and prevent wounds from infections. All the experimental results suggest that the hydrogel of PVP-CMC-BA will be an ideal wound dressing material compared to PVP-CMC.

\section{ACKNOWLEDGMENTS}

The authors are thankful to the Ministry of Education, Youth and Sports of the Czech Republic (MSM 70088352101) for providing the financial support. They are also thankful to Ms. Ludmila Zalesakova for help in lyophilized sample preparation for SEM and Ing. Michal Máchovský for taking SEM images.

\section{REFERENCES}

[1] Park, K.R., and Nho, Y.C. (2003) Synthesis of PVA/PVP hydrogels having two-layer by radiation and their physical properties. Radiat. Phys. Chem., 67:361-365.

[2] Wang, M., Xu, L., Hu, H., Zhai, M., Peng, J., Nho, Y., Li,J., and Wei, G. (2007) Radiation synthesis of PVP/CMC hydrogels as wound dressing. Nucl. Instrum. Meth. B., 265:385-389.

[3] Sen, M., and Avci, E.N. (2005) Radiation synthesis of poly (iV-vinyl-2-pyrrolidone)-Kr carrageenan hydrogels and their use in wound dressing applications. I. Preliminary laboratory tests. J. Biomed. Mater. Res., 74 A:187-196.

[4] Hydrogel burn and injury dressing, http://www.dae.gov.in/publ/betrlife/health/hydrogel.pdf / data. 2010.

[5] Rosiak, J., Rucinska-rybas, A., and Pekala, W. (1989) Method of manufacturing hydrogel dressings. U.S. Patent Application 4871490.

[6] Higa, O.Z., Rogero, S.O., Machado, L.D.B., Mathor, M.B., and Lugao, A.B. (1999) Biocompatibility study for PVP wound dressing obtained in deterrent conditions. Radiat. Phys. Chem., 55:705-707.

[7] Benamer, S., Mahlous, M., Boukrif, A., Mansouri, B., and Youcef, S.L. (2006) Synthesis and characterisation of hydrogels based on poly (vinyl pyrrolidone). Nucl. Instrum. Meth. B., 248:284-290.

[8] Kutsenko, L.I., Santuryan, Y.G., Karetnikova, E.B., Gofman, I.V., Bochek, A.M., and Panann, E.F. (2007) Properties of the methyl cellulose-polyvinylpyrrolidone binary system in solution and in the solid state. Macromol. Chem. Polym. Mater., 80:771-776.

[9] Zhao, L., Xu, L., Mitomo, H., and Yoshii F. (2006) Synthesis of pH-sensitive PVP/CM-chitosan hydrogels with improved surface property by irradiation. Carbohyd. Pólym. 64:473-480.

[10] Bagnas, M.P. (2005). Study of water diffusion through hydogels. Published on Graduate College at Illinois (http://www.grad.uiuc.edu).

[11] Lyon, J.G., Geever, L.M., Nugent, M.J.D., Kennedy, J.E., and Higginbotham C.L. (2009) Development and characterisation of an agar-polyvinyl alcohol blend hydrogel. J. Mech. Behav. Biomed. Matter. 2:485-493.

[12] Boric acid, http://www.buzzle.com/articles/boric-acid.html/data. 2010

[13] Boroline, http://www.boroline.com/boroline.php/data. 2010. 
[14] Desistores: Network of Indian Stores, http://www.desistores.net/catalog/product_info.phpPproducts id $=1208 \&$ osCsid=4bd56f $2 \mathrm{fa} 2 e e 144 \mathrm{a} 86 \mathrm{dc} 07 \mathrm{f} 2856 \mathrm{~cd} 20 \mathrm{c} / \mathrm{data} .2010$.

[15] B-TEX ointment and lotion, http://www.btex.in/btexwhite.html/data. 2010

[16] Toxicological review of boron and compounds (GAS No. 7440-42-8), In support of summary Information on the integrated risk information system (IRIS), June 2004. U.S. Environmental Protection Agency, Washington, DC.

[17] Boric acid health and safety datasheet, http://www.etiproducts.com/files/MSDS__Boric_Acid.pdf / data. 2010

[18] El-Mohdy, H.L.A., and Safrany, A. (2008) Preparation of fast response superabsorbent hydrogels by radiation polymerization and crosslinking of N-isopropylacrylamide in solution. Radiat. Phys. C/im.77:273-279.

[19] Rosiak,J.M., Ulanski, P., Pajewski, L.A., Yoshii, F., and Makuuchi, K. (1915) Radiation formation of hydrogels for biomedical purposes. Some remarks and comments. Radiat. Phys. Chem.46 (2):161-168.

[20] IAEA-RCA workshop on radiation processing of natural polymers for healthcare applications, Bhaba Atomic Research Centre, India, November 3-7.

[21] Ghosh, A., Ali, M.A., and Dias, G.J. (2009) Effect of cross-linking on microstructure and physical performance of casein protein. Biomacromolecules, 10:1681-1688.

[22] Saha, P., Saha, N., and Roy, N. (2008) Hydrogel Wound Covering, Patent filed at Czech patent office (UPV CR). File number PV 2008-306.

[23] Nishida, T., Nakata, K., and Nakamura, M. (2006) Skin wound healing promoters, U.S. Patent 7071166.

[24] Barth, J.B. (1976) Humectant sweetener, U.S. Patent 3932604

[25] Schacht, E.H. (2004) Polymer chemistry and hydrogel systems./. Phys. Conf. Ser., 3: 22-28.

[26] What is boric acid? www.rosemill.com/html/boric_acid_information.pdf/data. 2010.

[27] Barbucci, R., Magnani, A., and Consumi, M. (2000) Swelling behavior of carboxymethylcellulose hydrogels in relation to cross-linking, pH, and charge density. Macromolecules, 33:7475-7480.

[28] Pal, K., Banthia, A.K., and Majumdar, D.K. (2007) Preparation and characterization of polyvinyl alcohol-gelatin hydrogel membranes for biomedical applications. AAPS. PharmSciTech., 8(1): Article 21.

[29] Saraydin, D., and Caldiran, Y. (2001) In vitro dynamic swelling behaviors of polyhydroxamic acid hydrogels in the simulated physiological body fluid. Polym. Bull., 46:91-98.

[30] Deng. C., He, L., Zhao, M., Yang, D., and Liu, Y. (2007) Biological properties of the chitosan- gelatin sponge wound dressing. Carbohyd. Polym., 69:583-589.

[31] Galya, T., Sedlarik, V., Kuritka, I., Novotny, R., Sedlarikova, J., and Saha, P. (2008) Antibacterial poly (vinyl alcohol) film containing silver nanoparticles: Preparation and characterization./. Appl. Polym. Sci, 110:3178-3185.

[32] Pan, Y.S., Xiong, D.S., and Ma, R.Y. (2006) Preparation and swelling behavior of polyvinyl alcohol physiological saline gel./ Cent. South Univ. Technol, 13(1). Article ID: 1005 “ 9784(2006) 01” 0027 “05.

[33] Horkay, F., Tasaki, I., and Basser, P.J. (2000) Osmotic swelling of polyacrylate hydrogels in physiological salt solutions. Biomacromolecules., 1:84-90.

[34] Ohishi, K., Itadani, T., Hayashi, T., Nakai, T., and Horii, F. (2010) Role of boric acid in the formation of poly (vinyl alcohol)-iodine complexes in undrawn films. Polymer., 51:687-693.

[35] Rosiak, J.M., and Ulanski, P. (1999) Synthesis of hydrogels by irradiation of polymers in aqueous solution. Radiat. Phys. Chem., 55:139-151.

[36] Roy, N., Saha, N., Kitano, T., and Saha, P. (2009) Effect of swelling on rheological behavior of hydrogels in Proceedings of 25th Annual Meeting of the Polymer Processing Society, Goa, India, March 1-5.

[37] Weng, L., Chen, X., and Chen, W. (2007) Rheological characterization of in situ crosslinkable hydrogels formulated from oxidized dextran and N-Carboxyethyl chitosan. Biomacromolecules., 8:1109-1115.

[38] Zandraa, O., Saha, N., Pavlinek, V., Kitano, T., and Saha, P. (2008) Comparative study of diagnostic and therapeutic medical ultrasound gels in Proceedings ofPPS-24 Annual Meeting, Salerno, Italy, June 15-19.

[39] Mourtas, S., Haikou, M., Theodorpoulou, M., Tsakiroglou, C., and Antimisiaris, S.G. (2008) The effect of added liposomes on the rheological properties of a hydroerel: A systematic study. J. Colloid. Interf. Sci, 317:611-619. 